\title{
Novel Mutation-Deletion in the PHOX2B Gene of the Patient Diagnosed with Neuroblastoma, Hirschsprung's Disease, and Congenital Central Hypoventilation Syndrome (NB-HSCR- CCHS) Cluster
}

Izabela Szymońska', Thore Langfeldt Borgenvik' ${ }^{2}$, Tina Margrethe Karlsvik², Anders Halsen ${ }^{2}$, Bianka Kathryn Malecki ${ }^{2,5}$, Sindre Ervik Saetre ${ }^{2}$, Mateusz Jagła', Piotr Kruczek', Anna Madetko Talowska ${ }^{3}$, Grażyna Drabik ${ }^{4}$, Magdalena Zasada', Marek Malecki ${ }^{5,6,7 *}$

'Department of Pediatrics, Jagiellonian University Medical College, Krakow, Poland, EU

${ }^{2}$ Jagiellonian University Medical College, Krakow, Poland, EU

${ }^{3}$ Department of Clinical Genetics, Jagiellonian University Medical College, Krakow, Poland, EU

${ }^{4}$ Department of Pathology, Children's University Hospital, Kraków, Poland, EU

${ }^{5}$ Phoenix Biomolecular Engineering Foundation, San Francisco, CA, USA

${ }^{6}$ NMRFM, National Institutes of Health, Madison, WI, USA

${ }^{7}$ University of Wisconsin, Madison, WI, USA

\begin{abstract}
Introduction: Neuroblastoma (NB), Hirschsprung disease (HSCR), Congenital Central Hypoventilation Syndrome (CCHS), clinically referred as the NB-HSCR-CCHS cluster, is genetic disorders linked to mutations in the PHOX2B gene on chromosome $4 \mathrm{p} 12$.
\end{abstract}

Specific aim: The specific aim of this project is to define the $P H O X 2 B$ gene mutations as the genomic basis for the clinical manifestations of the NB-HSCR-CCHS cluster.

Patient: A one day old male patient presented to the JUMC neonatal ICU due to abdominal distention, vomiting, and severe apneic episodes. With the preliminary diagnosis of the NB-HSCR-CCHS, the blood and tissue samples were acquired from the child, as well as from the child's parents. All procedures were pursued in accordance with the Declaration of Helsinki, with the patient's Guardian Informed Consent and the approval from the Institutional Review Board.

Genetic/genomic methods: Karyotyping was analyzed based upon Giemsa banding. The patient's genomic DNA was extracted from peripheral blood and amplified by polymerase chain reaction. Direct microfluidic Sanger sequencing was performed on the genomic DNA amplicons. These procedures were pursued in addition to the routine clinical examinations and tests.

Results: G-banding showed the normal $46 \mathrm{XY}$ karyotype. However, genomic sequencing revealed a novel, heterozygous deletion (8 nucleotides (c.699-706, del8) in exon 3 of the PHOX2B gene on chromosome 4. This led to the frame-shift mutation and malfunctioning gene expression product.

Conclusion: Herein, we report a novel PHOX2B gene mutation in the patient diagnosed with the NB-HSCR$\mathrm{CCHS}$ cluster. The resulting gene expression product may be a contributor to the clinical manifestations of these genetic disorders. It adds to the library of the mutations linked to this syndrome. Consequently, we suggest that screening for the PHOX2B mutations becomes an integral part of genetic counseling, prenatal screening, and preparing supportive therapy upon delivery.

Keywords: Neuroblastoma (NB); Hirschsprung's disease (HSCR); Congenital central hypoventilation syndrome (CCHS); NB-HSCRCCHS; Aganglionosis of the terminal bowel (ATB); Neurocristopathy, haddad syndrome, $P H O X 2 B$, SOX10, 4p12; Mutation; Deletion

Abbreviations: CCHS: Congenital Central Hypoventilation Syndrome; HSCR: Hirschsprung Disease; ATB: Aka Aganglionosis of the Terminal Bowel; NB: Neuroblastoma; SIDS: Sudden Infant Death Syndrome; PHOX2B: Paired-like Homeobox 2b

\section{Introduction}

Congenital Central Hypoventilation Syndrome (CCHS) is a breathing disorder caused by a decreased sensitivity to hypercapnia and hypoxia in the brainstem. This leads to hypoventilation, especially during non-REM sleep in most patients, but also during wakeful states in more severely affected individuals. Patients with CCHS often present with shallow breathing leading to cyanosis within the first hours of life, but can in milder cases go undiagnosed for years. Due to the carbon dioxide retention that frequently develops in these patients, breathing support or mechanical ventilation is often necessary [1-6].
Hirschsprung's Disease (HSCR) is a motor disorder of the gastrointestinal tract [7]. It is caused by failure of neural crest cells to properly migrate during intestinal development. The aganglionic intestinal segment is unable to relax, which leads to functional obstruction [8-11].

*Corresponding author: Marek Malecki, M.D, Ph.D, Phoenix Biomolecula Engineering Foundation, San Francisco, CA, USA, Tel: 4157134370; Fax: 4157134371; E-mail: mm@PBMEF.org

Received July 14, 2015; Accepted September 01, 2015; Published September 07, 2015

Citation: Szymońska I, Borgenvik TL, Karlsvik TM, Halsen A, Malecki BK, et al. (2015) Novel Mutation-Deletion in the PHOX2B Gene of the Patient Diagnosed with Neuroblastoma, Hirschsprung's Disease, and Congenital Central Hypoventilation Syndrome (NB-HSCR- CCHS) Cluster. J Genet Syndr Gene Ther 6: 269. doi:10.4172/2157-7412.1000269

Copyright: (c) 2015 Szymońska I, et al. This is an open-access article distributed under the terms of the Creative Commons Attribution License, which permits unrestricted use, distribution, and reproduction in any medium, provided the original author and source are credited. 
Citation: Szymońska I, Borgenvik TL, Karlsvik TM, Halsen A, Malecki BK, et al. (2015) Novel Mutation-Deletion in the PHOX2B Gene of the Patient Diagnosed with Neuroblastoma, Hirschsprung's Disease, and Congenital Central Hypoventilation Syndrome (NB-HSCR- CCHS) Cluster. J Genet Syndr Gene Ther 6: 269. doi:10.4172/2157-7412.1000269

Page 2 of 6

Neuroblastoma (NB) is a neuroendocrine tumor that arises from neural crest cells. It is the most common neoplasm in infancy and the most common extracranial solid cancer in childhood. The vast majority of cases are non-familial. However, in rare cases of familial neuroblastomas, they have been shown to carry mutations in Anaplastic Lymphoma Kinase (ALK), Paired-like homeobox 2b (PHOX2B), and KIF1B genes [12-14].

During fetal development a reciprocal balance between expression of $P H O X 2 B$ and SOX10 is responsible for maintaining the processes of neuronal and glial differentiation of the neural crest. Central Congenital Hypoventilation Syndrome (CCHS), Hirschsprung's Disease (HSCR) and Neuroblastoma (NB) are categorized as neurocristopathies [1521]. These are genetic disorders caused by aberrant development of neural crest cells. $90 \%$ of the patients with CCHS are heterozygous for mutations in exon 3 of the paired-like homeobox 2B gene, (PHOX2B) on chromosome 4 . It is responsible for encoding the transcription factor required for neuronal development. Collectively, they are often referred to as the NB-HSCR-CCHS cluster [22]. Interestingly, Sudden Infant Death Syndrome (SIDS) has also been recently linked to this gene's mutations.

In healthy individuals, the $P H O X 2 B$ gene has an exon coding for 20 alanine repeat sequence. Gene mutations, that expand this region, are known as polyalanine repeat mutations (PARMs). They are responsible for over $90 \%$ of $P H O X 2 B$ mutations. Alternatively, missense, nonsense, or frameshift mutations in the $P H O X 2 B$ gene result in non-polyalanine repeat mutations (nPARMs). While these mutations account for less than $10 \%$ of cases, they lead to a more severe phenotype and most aggravated clinical manifestations.

Herein, we describe a novel nPARMs - mutation deletion in exon 3 (c.699-706, del8) of the PHOX2B gene located on the chromosome $4 \mathrm{p} 12$, in the male neonate, who has been clinically diagnosed with the CCHS, HSCR and NB, also referred as the NB-HSCR-CCHS cluster.

\section{Patient's Clinical Presentation}

A $2900 \mathrm{~g}$ male was born at 41 weeks gestation by vaginal delivery on December 4, 2010, to a 24 years old Gravida II Para II mother. The boy appeared healthy at birth with APGAR scores of 5 and 8 at the first and fifth minute, respectively. The pregnancy was complicated by polyhydramnios.

The patient was admitted to the Jagiellonian University Medical Center (JUMC) American Children's Hospital in Prokocim, Poland, European Union, on the second day of his life (Figures A-F). The parents were presented with the Patient's Bill of Rights in accordance with the Declaration of Helsinki and signed the Guardian Informed Consent. All the procedures that followed were approved by the Institutional Review Board.

Prenatal screening was offered in the next pregnancy, but was declined. The male newborn, Gravida III Para III, has shown no symptoms of neurocristopathies.

\section{Materials and Methods}

\section{Ultrasonography (USG)}

Ultrasonography was performed using the EnVisor C; Philips Medical Systems Nederland BV (Best, the Netherlands). The probe used: was Micro convex at the frequency $10 \mathrm{MHz}$.

\section{$\mathrm{X}$-ray and computed tomography $(\mathrm{CT})$}

$\mathrm{X}$-ray imaging followed by Computed Tomography (CT) were pursued on the General Electric Light Speed VCT. The contrast agent administered for CT was Ultravist 300 at $2-3 \mathrm{ml} / \mathrm{kg}$. The images were analyzed using Advantage Windows software from the General Electric (Milwaukee, WI, USA).

\section{Capillary electrophoresis}

Analysis was performed on the Next Generation Capillary Electrophoresis System: The 3500 Series Genetic Analyzers ABI 3500 (Life Technologies, Waltham, MA, USA, Cat\#: 4404312).

\section{Histopathology}

Multiple intestinal biopsies were taken. The samples were fixed in buffered formalin solution. They were dehydrated and embedded in paraffin. The tissues in paraffin blocks were cut into 3 micrometer thick sections. For patomorphology, the sections were stained with Hematoxylin and Eosin. For immunocytochemistry, the sections were labeled with antibodies against Neuronal Specific Enolase (DAKO) followed by reporters: HRP based EnVision+ System with $\mathrm{DAB}+$ Substrate-Chromogen (DAKO). The immunolabelled sections were also counterstained with Hematoxylin and Eosin. The samples were mounted and photographed.

\section{Blood tests}

Blood for routine testing was drawn upon the admission to the hospital and followed up daily according to the sterile clinical procedures. Blood was also evaluated for neuron specific enolase and ferritin.

\section{Urine tests}

Concentrations of catecholamines in urine were determined by electrocheminal detection on high pressure liquid chromatography (HPLC).

\section{Karyotyping}

$2 \mathrm{ml}$ of blood was collected into tubes with Heparin (Vacucol Lithium Heparin, Medlab Products). Then it was cultured for 72 $\mathrm{h}$ in the incubator (Heraeus, Hera cell, Germany, EU). The culture medium consisted of $2 \mathrm{ml}$ of the Fetal Calf Serum (Biowest, South America), $8 \mathrm{ml}$ of RPMI (Wytwórnia Surowic i Szczepionek Biomed, Lublin, Poland, EU) and 0,8 $\mathrm{ml}$ of LF-7 (Biomed, Krakow, Poland, EU). For synchronization, after 72 h, 4 drops of colchicine was added (KaryoMAX COLCEMID Solution, Gibco, Grand Island, New York, USA). The cultures were centrifuged for 8 minutes at $1200 \mathrm{rpm}$ at the room temperature. The supernanatant was discarded. The cells were resuspended in $3 \% \mathrm{KCl}$ and incubated for 30 minutes at 30 degrees C. The cells were fixed in the 3:1 mixture of acetic acid and methanol (Chempur, Piekary Silesia, Poland and EU). The slide carrying the chromosomes were immersed in a preparation of trypsin preparation $\left(0.16 \mathrm{~g}\right.$ of trypsin dissolved in $100 \mathrm{ml}$ of $\mathrm{KH}_{2} \mathrm{PO}_{4}$ and $\mathrm{Na}_{2} \mathrm{HPO}_{4} \times 12$ $\mathrm{H}_{2} \mathrm{O}$, mixed in the ratio $\left.1: 1\right)$ for one second, and then rinsed in icewater. The samples were stained for $1 \mathrm{~min}$ with Wright's eosin methylene blue solution and sealed with mounting medium. [23] The mounts were recorded with the Nikon microscope.

\section{Genomics}

The genomic DNA was extracted from peripheral blood with the use of a Master Pure DNA Purification Kit (Epicentre-Illumina, Eindhoven, NL, EU, Cat\#: MCD85201) according to the manufacturer's instructions. Polymerase chain reaction based amplification was performed according to the guidelines facilitating down-stream Sanger Sequencing $[24,25]$. 
Citation: Szymońska I, Borgenvik TL, Karlsvik TM, Halsen A, Malecki BK, et al. (2015) Novel Mutation-Deletion in the PHOX2B Gene of the Patient Diagnosed with Neuroblastoma, Hirschsprung's Disease, and Congenital Central Hypoventilation Syndrome (NB-HSCR- CCHS) Cluster. J Genet Syndr Gene Ther 6: 269. doi:10.4172/2157-7412.1000269

Sanger sequencing (Life technologies Sanger sequencing workflow) was performed on the amplicons after PCR with the use of a Big Dye Terminator Kit (Life Technologies-Analytik, Warsaw, Poland, EU, Cat\#: 4337455).

Sequencing primers: The primers were designed based upon the GenBank sequence using the National Institutes of Health GST Prime software (Bethesda, MD, USA) and synthesized by Genomed S.A (Warsaw, Poland, EU) (Table 1).

\section{Results}

Upon the admission, the neonate patient presented with generalized muscular hypotonia and dysmorphic facial features, including downward-slanting palpebral fissures, prominent forehead, and hypoplastic facial bones with retro- and micrognathia. The patient failed to pass meconium and presented with abdominal distention, vomiting and greenish gastric residuals (Figure 1).

Upon the initial screening, findings of cranial and abdominal ultrasound were unremarkable. Radiologic examination suggested intestinal atresia or long segment HSCR. Furthermore, serial abdominal $\mathrm{X}$-rays revealed dilated proximal intestinal loops with a microcolon (Figure 2). A barium enema contrast study showed microcolon.

The infant underwent an open laparotomy with resection of the colon and formation of an ileostomy.

\begin{tabular}{|l|c|}
\hline Forward primer & 5'ctgcttcaccgtctctccttcc \\
\hline Reverse primer & 5'-tacccgctcgcccactcg \\
\hline
\end{tabular}

Table 1: PCR primer sequences.
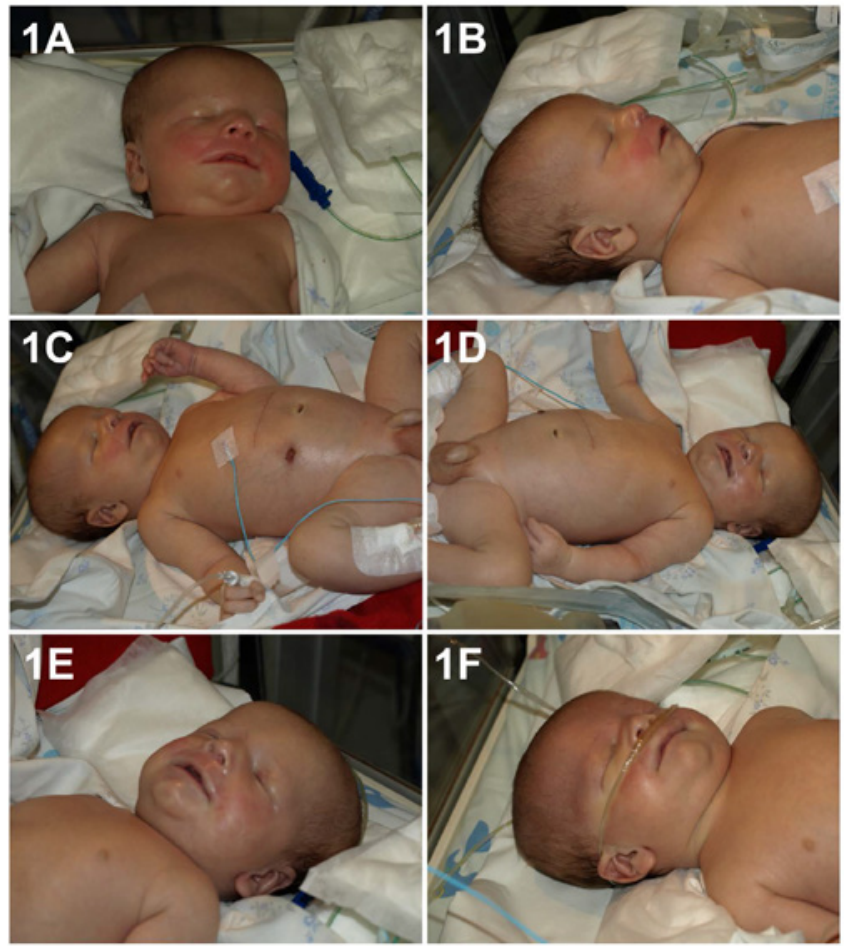

Figure 1: A-B. The facial features of the patient. Notice downward-slanting palpebral fissures, prominent forehead, and hypoplastic facial bones with retroand micrognathia. C-F. The overall appearance of the patient. Notice the scars after laparotomy.

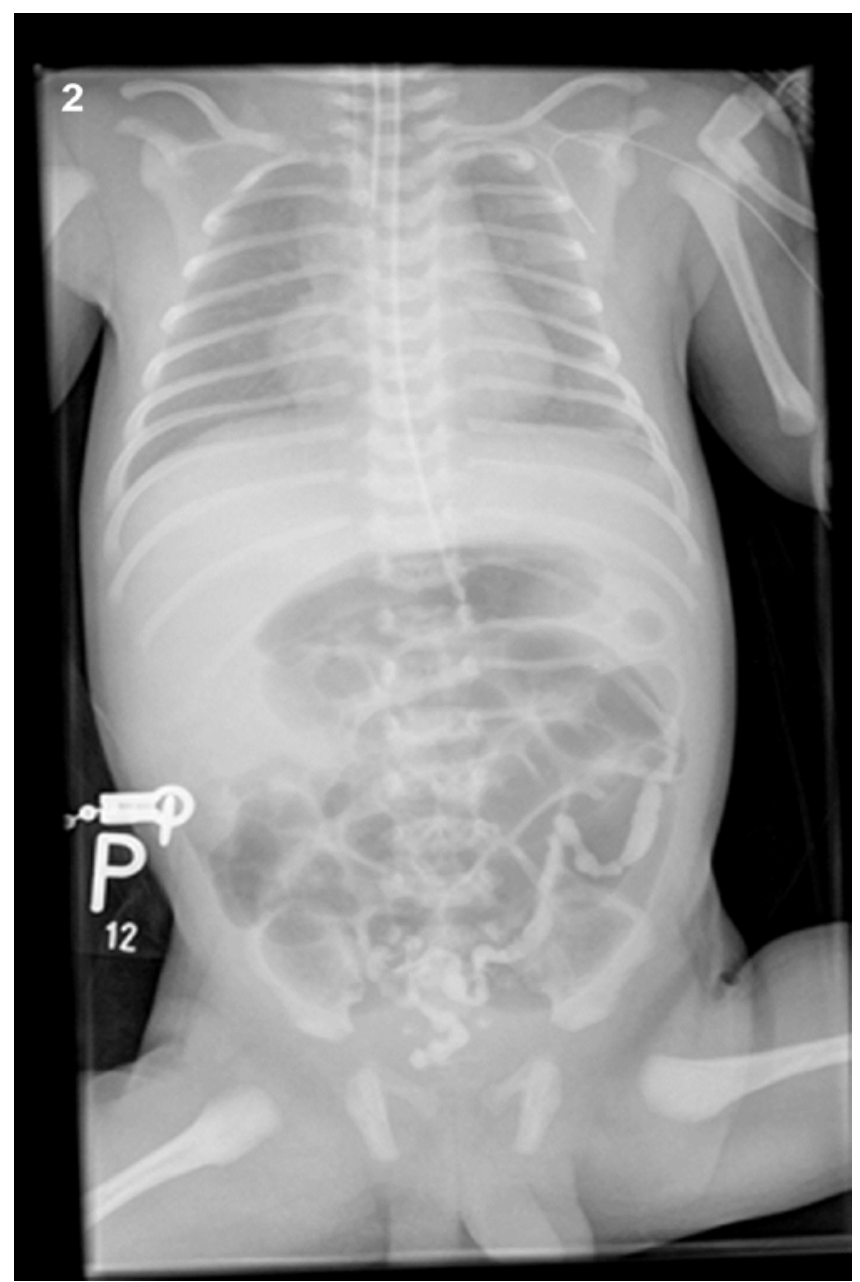

Figure 2: A projection from the abdominal X-ray performed after the patient's first laparotomy. It reveals the small bowel distention and microcolon.

The tissue samples were acquired during the first laparotomy. The microscopic examination of the resected colon showed aganglionosis of the entire intestinal tract. This resulted in the clinical diagnosis of Hirschsprung's Disease (HSCR).

The specific tests for neuronal specific enolase in serum revealed its increased level: $40.37 \mathrm{ng} / \mathrm{ml}$. The value for ferritin reached $487.2 \mu \mathrm{g} / \mathrm{l}$.

Urine was collected daily for measurements of the catecholamines' metabolites, as further metanephrine and normetanephrine are both metabolized to vanillylmandelic acid. These values were increasingly elevated in consecutive days: 16.295; HVA: 32.993; 5HIAA: 22.836; Normetanephrine: $1.851 \mathrm{mcg} / \mathrm{mg}$ creatinine $(121-946 \mathrm{mcg} / \mathrm{g}$ creatinine); Metanephrine: $0.219 \mathrm{mcg} / \mathrm{mg}$ creatinine $(82-418 \mathrm{mcg} / \mathrm{g}$ creatinine); 3 Metoksytyramine: $0.621 \mathrm{mcg} / \mathrm{mg}$ creatinine. These results furthered the diagnosis of Neuroblastoma.

Due to recurrent intestinal obstruction and an aggravated clinical status, CT scans were performed. They showed two calcified tumor masses located bilaterally in the thoracolumbar paravertebral area, and one calcified mass in the left suprarenal area extending from the renal vessels to the abdominal aorta (Figure 3). The ROI liver scan revealed multiple foci of metastases.

The recurrent intestinal obstruction prompted the second 
Citation: Szymońska I, Borgenvik TL, Karlsvik TM, Halsen A, Malecki BK, et al. (2015) Novel Mutation-Deletion in the PHOX2B Gene of the Patient Diagnosed with Neuroblastoma, Hirschsprung's Disease, and Congenital Central Hypoventilation Syndrome (NB-HSCR- CCHS) Cluster. J Genet Syndr Gene Ther 6: 269. doi:10.4172/2157-7412.1000269

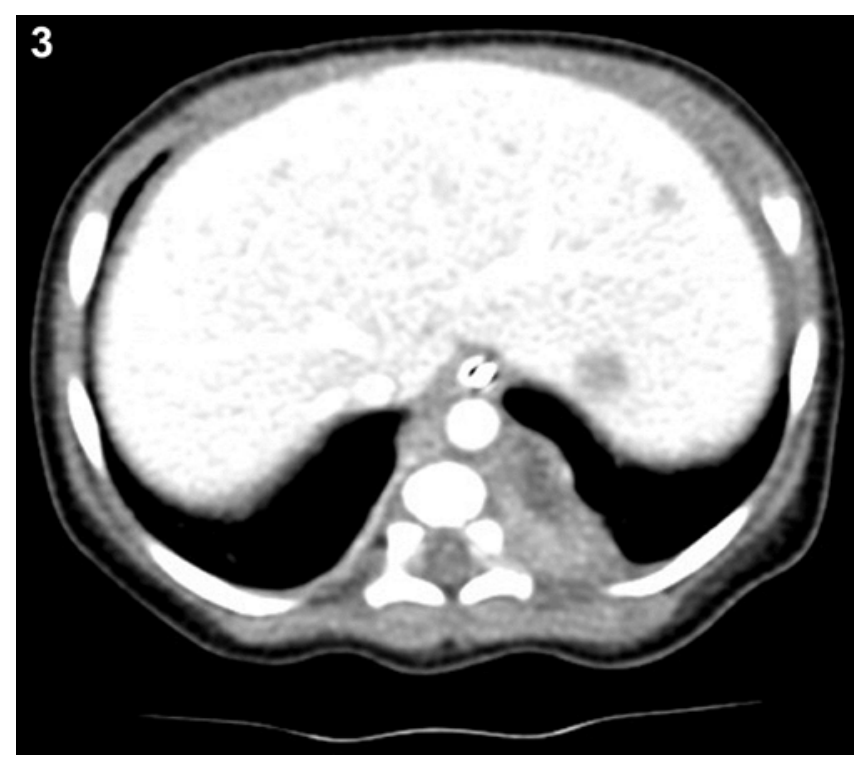

Figure 3: A section from the abdominal CT conducted during the third week of life. It highlighted calcified liver mass.

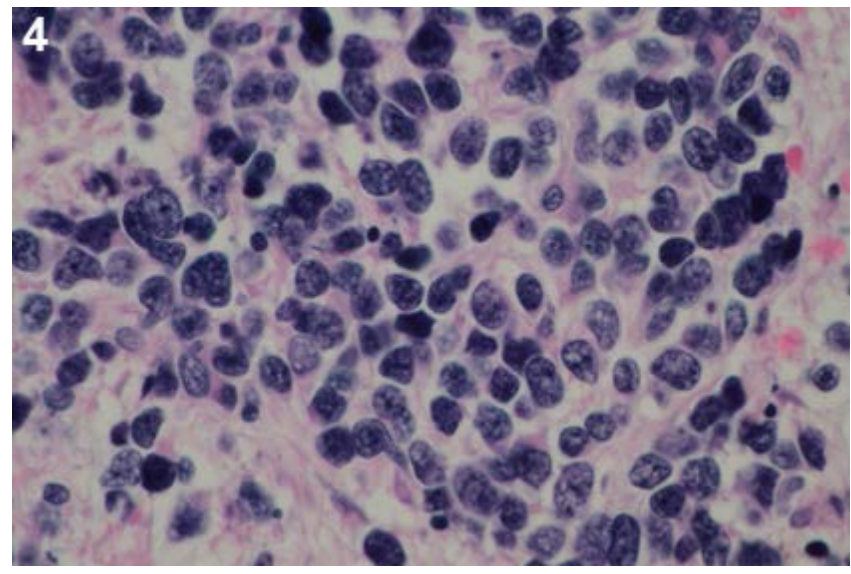

Figure 4: A microscopic image of the biopsy taken during the second laparotomy after sectioning and staining with Hematoxylin and Eosin. The image facilitates diagnosis of Neuroblastoma (Schwannian stroma poor) poorly differentiated subtype composed of undifferentiated neuroblastic cells with recognizable neuropil (primary magnification x 400).

laparotomy on the $17^{\text {th }}$ day of life. It was prompted by radiologic findings of abdominal, calcified masses.

During the surgery, the biopsies of the suprarenal tumor and liver masses were acquired for histopathology and immunocytochemistry. The results supported the diagnosis of Neuroblastoma (Schwannian stroma poor) poorly differentiated subtype (Figure 4).

The baby experienced two apneic episodes. These were managed with oxygen supplementation and nasal CPAP. On the third day of life, the infant was intubated and mechanical ventilation was started using Synchronized Intermittent Mandatory Ventilation (SIMV). During hospitalization the patient experienced transient episodes of hypoxia with saturation as low as $60 \%$ and hypercapnia reaching 145 $\mathrm{mm} \mathrm{Hg}$. Every attempt of extubation failed and resulted in severe hypoventilation; therefore ventilator dependence was assured.
Based upon the blood draw and cell culture propagation, karyotyping was performed. It revealed the normal 46XY karyotype with no alterations (Figure 5).

Read-out of the Sanger sequencing of the patient's and parent's genomic DNA was performed after nested PCR amplification and amplicons' sequencing (Figure 6). It shows the portions of exon 3 of the PHOX2B gene, c.689-711. The gene sequencing of the genomic DNA revealed a heterozygous deletion: 8 nucleotides (c.699-706, del8) in exon 3 of the PHOX $2 B$ gene on chromosome 4 . Therefore, it resulted in the frameshift mutation in the patient's DNA.

Prompted by discovery of the nPARM mutation in the $P H O X 2 B$ in the offspring, the blood samples were also acquired from the parents. The sequencing was also performed on the genomic DNA using the same methodology, as for the patient. The results indicated that the parents had the wild type PHOX2B. Therefore, it was concluded that the deletion present in the patient, occurred de novo.

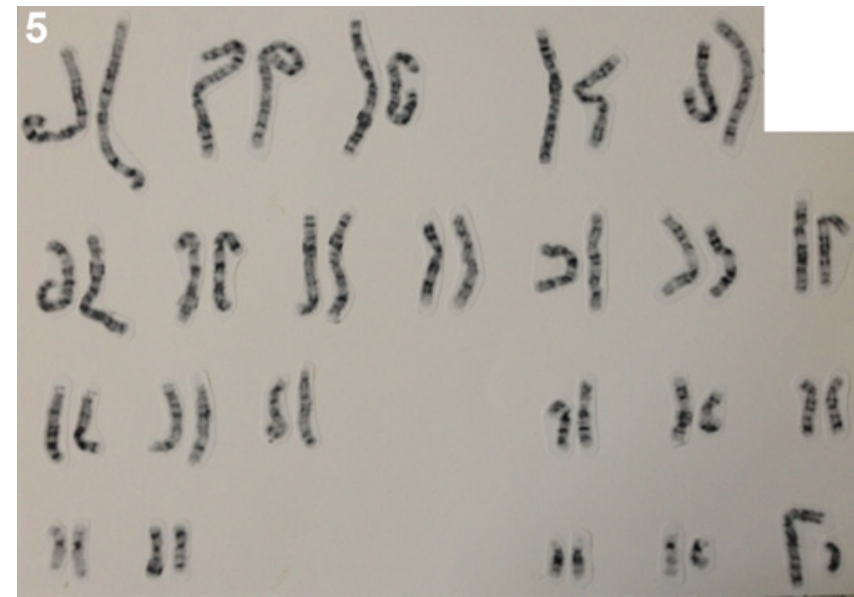

Figure 5: Chromosomes' spreading revealed the normal 46XY karyotype with no alterations. The patient's information has been suppressed (upper right corner).

a : : 2 :
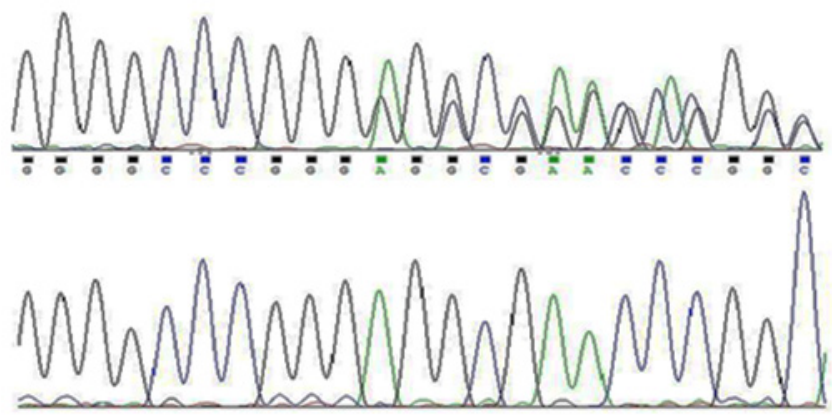

Figure 6: Read-out of the Sanger sequencing of the patient's and parent's genomic DNA. It shows the portions of exon 3 of the PHOX2B gene, c.689711.

Top: the sequence of the patient's PHOX2B gene revealing the heterozygous frameshift mutation c.699-706 del 8 (genomic sequence accession number ENSG00000109132). 
Citation: Szymońska I, Borgenvik TL, Karlsvik TM, Halsen A, Malecki BK, et al. (2015) Novel Mutation-Deletion in the PHOX2B Gene of the Patient Diagnosed with Neuroblastoma, Hirschsprung's Disease, and Congenital Central Hypoventilation Syndrome (NB-HSCR- CCHS) Cluster. J Genet Syndr Gene Ther 6: 269. doi:10.4172/2157-7412.1000269

Page 5 of 6

\section{Gene's Summary}

\section{Gene: $\mathrm{PHOX} 2 B$}

Description: Paired-like homeobox 2b [Source: HGNC Symbol; Acc: HGNC: 9143]

\section{Synonyms: NBPhox, $P H O X 2 B, P M X 2 B$}

Location: Chromosome 4: 41,744,082-41,748,970 reverse strand
INSDC
coordinates:
Chromosome:
GRCh38:

\section{CM000666.2:41744082:41748970:1}

About this gene: This gene has 2 transcripts (splice variants), 55 orthologues, 14 paralogues, is a member of 1 Ensembl protein family and is associated with 7 phenotypes.

\section{CCDS: This gene is a member of the Human CCDS set: CCDS3463.1}

UniProtKB: This gene has proteins that correspond to the following Uniprot identifiers: Q99453

RefSeq: Overlapping RefSeq Gene ID 8929 matches and has similar biotype of protein coding

LRG: LRG_513 provides a stable genomic reference framework for describing sequence variations for this gene

\section{Ensembl version: ENSG00000109132.6}

GRCh37 assembly: This gene maps to $41,746,099-41,750,987$ in GRCh37 coordinates.

\section{View this locus in the GRCh37 archive: ENSG00000109132 \\ Gene type: Known protein coding}

\section{Clinical Epilogue}

Due to the exceedingly poor prognosis, after ethical considerations, parents made the decision not to initiate invasive cancer therapy.

The patient was kept sedated on breathing support until he died of cardiac arrest on the $50^{\text {th }}$ day of his life.

\section{Discussion}

The $P H O X 2 B$ gene encodes a highly conserved homeodomain transcription factor important in the development and migration of neural crest cells. Mutations in this gene may lead to a variety of autonomic nervous system defects, including CCHS, HSCR and NB, as seen in our patient. More than $90 \%$ of patients with CCHS will be heterozygous for a mutation in this gene, while polyalanine repeat expansion mutations (PARMs) account for over $90 \%$ of them. A larger number of polyalanine repeats correlates with a more severe clinical phenotype, with the constellation of both CCHS and HSCR commonly being caused by an expansion of +6 or +7 alanines. A +5 PARM is being described as prevalent in the patient population diagnosed with late-onset central hypoventilation syndrome. The remaining PHOX2B mutations are classified as non-PARMs, including missense, nonsense and frameshift mutations, which are almost all de novo mutations with rare exceptions. This has been reported to result in a more severe phenotype, and is much more frequently associated with neuroblastomas.

Depending on the type of mutation involved, the clinical presentation of $P H O X 2 B$ mutations can range from being completely asymptomatic at birth, to severe $\mathrm{CO}_{2}$ retention, as seen in our patient. Other patients may present at an older age, with cyanosis, edema, and signs of right- sided heart failure as the first indications of CCHS. Still, others may present with unexplained apnea or an apparent life-threatening event, or some may even die and be categorized as having sudden infant death syndrome (SIDS). Late-onset central hypoventilation syndrome has also been described, for which symptoms present in late childhood or adulthood. The heterogeneity in expression and clinical presentation creates great difficulties in correctly diagnosing the $P H O X 2 B$ mutations, which may lead to delays in the initiation of proper treatment. Given the extreme clinical variability, it is possible that the prevalence of congenital central hypoventilation syndrome in the general population is much higher than previously estimated.

Futuristically thinking, since this single gene's mutations are linked to many genetic disorders, then potentially the $P H O X 2 B$ gene may become an ideal target for gene therapy trials.

\section{Conclusion}

Herein, we report a novel mutation in the $P H O X 2 B$ gene in the patient diagnosed with the NB-HSCR-CCHS cluster. The resulting gene expression product may be a contributor to the clinical manifestations of these genetic disorders. It adds to the library of the mutations linked to this syndrome. Consequently, testing for the PHOX2B mutation could be performed on circulating fetal cells ahead of delivery and should be performed in intubated infants, when breathing difficulties occur upon extubation. Therefore, we suggest that screening for a PHOX2B mutation could become integral parts of genetic counseling, prenatal screening, and preparing supportive therapy upon delivery.

\section{Acknowledgments}

This study was supported in part by the grants from the Phoenix Foundation and the National Institutes of Health.

\section{Conflict of Interest Statement}

The authors state no conflict of interest.

\section{References}

1. Windisch W, Hennings E, Storre JH, Matthys H, Sorichter S (2004) Long-term survival of a patient with congenital central hypoventilation syndrome despite the lack of continuous ventilatory support. Respiration 71: 195-198.

2. Weese-Mayer DE, Rand CM, Berry-Kravis EM, Jennings LJ, Loghmanee DA et al. (2009) Congenital central hypoventilation syndrome from past to future: Model for translational and transitional autonomic medicine. Pediatr Pulmono 44: $521-535$

3. Weese-Mayer DE Berry-Kravis EM, Ceccherini I, Keens TG, Loghmanee DA, et al. (2010) An official ATS clinical policy statement: Congenital centra hypoventilation syndrome: genetic basis, diagnosis, and management. Am J Respir Crit Care Med 181: 626-644.

4. Trochet D, O'Brien LM, Gozal D, Trang H, Nordenskjöld A et al. (2005) $P H O X 2 B$ genotype allows for prediction of tumor risk in congenital central hypoventilation syndrome. Am J Hum Genet 76: 421-426.

5. Berry-Kravis EM, Zhou L, Rand CM, Weese-Mayer DE (2006) Congenita central hypoventilation syndrome: $P H O X 2 B$ mutations and phenotype. Am J Respir Crit Care Med 174: 1139-1144.

6. Fine-Goulden MR, Manna S, Durward A (2009) Cor pulmonale due to congenital central hypoventilation syndrome presenting in adolescence. Pediatr Crit Care Med 10: e41-e42.

7. Alves MM, Sribudiani Y, Brouwer RW, Amiel J, Antiñolo G, et al. (2013) Contribution of rare and common variants determine complex diseasesHirschsprung disease as a model. Dev Biol 382: 320-329.

8. Butler Tjaden NE, Trainor PA (2013) The developmental etiology and pathogenesis of Hirschsprung disease. Transl Res 162:1-15.

9. Musser MA, Michelle Southard-Smith E (2013) Balancing on the crest - Evidence for disruption of the enteric ganglia via inappropriate lineage segregation and consequences for gastrointestinal function. Dev Biol 382: 356-364. 
Citation: Szymońska I, Borgenvik TL, Karlsvik TM, Halsen A, Malecki BK, et al. (2015) Novel Mutation-Deletion in the PHOX2B Gene of the Patient Diagnosed with Neuroblastoma, Hirschsprung's Disease, and Congenital Central Hypoventilation Syndrome (NB-HSCR- CCHS) Cluster. J Genet Syndr Gene Ther 6: 269. doi:10.4172/2157-7412.1000269

Page 6 of 6

10. Goldstein AM, Hofstra RM, Burns AJ (2013) Building a brain in the gut: Development of the enteric nervous system. Clin Genet 83: 307-316.

11. Pan ZW, Li JC (2012) Advances in molecular genetics of Hirschsprung's disease. Anat Rec (Hoboken) 295: 1628-1638.

12. Pandey GK, Kanduri C (2015) Long noncoding RNAs and neuroblastoma. Oncotarget 6: 18265-18275.

13. Buhagiar A, Ayers D (2015) Chemoresistance, cancer stem cells, and miRNA influences: The case for neuroblastoma. Anal Cell Pathol (Amst) 2015: 1-8.

14. Louis CU, Shohet JM (2015) Neuroblastoma: molecular pathogenesis and therapy. Annu Rev Med 66: 49-63.

15. Pattyn A, Morin X, Cremer H, Goridis C, Brunet JF (1999) The homeobox gene $P H O X 2 B$ is essential for the development of autonomic neural crest derivatives. Nature 399: 366-370.

16. Trochet D, de Pontual L, Straus C, Gozal D, Trang H, et al. (2008) PHOX2B germline and somatic mutations in late-onset central hypoventilation syndrome. Am J Respir Crit Care Med 177: 906-911.

17. Liebrechts-Akkerman G, Liu F, Lao O, Ooms AH, van Duijn K, et al. (2014) PHOX2B polyalanine repeat length is associated with sudden infant death syndrome and unclassified sudden infant death in the Dutch population. Int $\mathrm{J}$ Legal Med 128: 621-629.
18. Mahfouz AKM, Rashid M, Khan MS, Reddy P (2011) Late onset congenital central hypoventilation syndrome after exposure to general anesthesia. Can $\mathrm{J}$ Anaesth 58: 1105-1109.

19. Lee P, Su Y-N, Yu C-J, Yang P-C, Wu H-D (2009) PHOX2B mutation-confirmed congenital central hypoventilation syndrome in a Chinese family: Presentation from newborn to adulthood. Chest 135: 537-544.

20. Parodi S, Vollono C, Baglietto MP, Balestri M, Di Duca M, et al. (2010) Congenital central hypoventilation syndrome: genotype-phenotype correlation in parents of affected children carrying a PHOX2B expansion mutation. Clin Genet 78: 289- 293.

21. Jennings LJ, Yu M, Rand CM, Kravis N, Berry-Kravis EM, et al. (2012) Variable human phenotype associated with novel deletions of the PHOX2B gene. Pediatr Pulmonol 47: 153-161.

22. http://www.ncbi.nlm.nih.gov/gene/8929

23. Bickmore WA (2012) "Karyotype Analysis and Chromosome Banding."

24. "Protocol for MasterPure ${ }^{\mathrm{TM}}$ Complete DNA - Epicentre." 2013.

25. "Applied Biosystems® | Life Technologies." 2013. <http://www.lifetechnologies. $\mathrm{com} / \mathrm{pl} / \mathrm{en} / \mathrm{home} /$ life-science/sequencing/sanger-sequencing/sanger-dnasequencing/pcr-sanger-sequencing.html> 